\title{
MOVIMIENTOS URBANOS Y COMUNICACION TRANSFORMADORA: ELEMENTOS DE ANÁLISIS DEL NUEVO ACTIVISMO DIGITAL
}

\author{
Dr. Francisco Sierra Caballero \\ Universidad de Sevilla, Sevilla, España \\ fsierra@us.es \\ ORCID iD: https://orcid.org/0000-0001-7398-7588
}

Recibido el 29 de mayo de 2019

Aceptado el 28 de octubre de 2019

\begin{abstract}
Resumen
La emergencia de nuevos procesos de participación local y global han redefinido el contexto social objeto de deliberación científica, apuntando la constatación de formas inéditas de acción colectiva. El nuevo netactivismo del derecho a la ciudad hace que el ejercicio de la ciudadanía y el buen gobierno sean concebidos como la construcción no solo de procesos de inclusión y deliberación ciudadana, sino sobre todo como un proceso de lucha y apropiación por recursos difusos como Internet, un proceso en fin de lucha por el código que exige una mayor permeabilidad y apertura cognitiva. Así, hoy asistimos a la proliferación de una nueva complejidad colectiva múltiple, y derivado de ello a una crisis de la representación, que demandan del pensamiento y la teoría social. La ciberdemocracia debe plantearse como una Economía Política del Archivo, como una crítica metacognitiva de la captura de la experiencia vivencial de la cibercultura, comenzando con los indicadores de inclusión digital y concluyendo con los modos de compartir y socializar el saber sobre lo social. En este marco es preciso plantear nuevos conceptos sobre la construcción del ágora virtual. El siguiente artículo aborda una revisión de la literatura especializada en la materia avanzando elementos para una crítica de los procesos tecnopolíticos contemporáneos a partir de un análisis estructural de los procesos de mediación y construcción colectiva de formas comunes de articulación de espacios autónomos por parte de los nuevos movimientos urbanos como resultado de la crisis de representación del régimen de mediación liberal.
\end{abstract}

Palabras clave: espacio público, tecnopolítica, teoría crítica, movimientos urbanos, ciberdemocracia. 


\title{
URBAN MOVEMENTS AND TRANSFORMING COMMUNICATION: ELEMENTS OF ANALYSIS OF THE NEW DIGITAL ACTIVISM
}

\begin{abstract}
The emergence of new processes of local and global participation has redefined the social context that is the object of scientific deliberation, pointing to the confirmation of unprecedented forms of collective action. The new netactivism of rights to the city means that exercising citizenship and good governance is conceived not only as the construction of processes of citizen inclusion and deliberation, but above all as a process of struggle and appropriation through diffuse resources such as the Internet, a process that finally fights for the code that demands greater permeability and cognitive openness. Thus, we are witnessing today the proliferation of a new multiple collective complexity, and derived from it a crisis of representation, which demands social thought and theory. Cyberdemocracy must be approached as a Political Economy of the Archive, as a metacognitive critique of the capture of the experience of cyberculture, beginning with the indicators of digital inclusion and ending with the ways of sharing and socializing knowledge about the social. Within this framework it is necessary to propose new concepts on the construction of the virtual agora. The following article deals with a review of specialized literature on the subject, advancing elements for a critique of contemporary technopolitical processes from a structural analysis of the processes of mediation and collective construction of common forms of articulation of autonomous spaces by the new urban movements as a result of the crisis of representation of the liberal mediation regime.
\end{abstract}

Keywords: public space, technopolitics, critical theory, urban movements, cyber democracy. 
Introducción

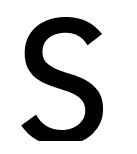

i la era de la cultura global es, en definición de Manuel Castells (1997), la era de la sociedad-red, dominada por el gobierno de las máquinas inteligentes, la investigación social debe redefinir nuevos puntos de anclaje, dando mayor relevancia a las dinámicas autónomas de la cultura digital que hoy redefinen las formas de mediación social de la cultura urbana, siendo como es la topología de esta posmodernidad radicalmente alterada por los relatos e intercambios de las imágenes de la ciudad neoliberal desde la cibercultura. Toda conceptualización teórica sobre el interfaz Ciudadanía/Nuevas Tecnologías de la Información debe, en coherencia, abordar en su radical singularidad, y desde el plano concreto de la inmanencia, el marco de conflictos y contradicciones que atraviesan los nuevos imaginarios urbanos, así como los procesos de acceso y apropiación local de la tecnocultura, considerando desde una visión crítica el papel de las políticas públicas, y las nuevas formas de dominio y control social que inaugura el actual modelo de desarrollo y reproducción social en las nuevas metrópolis uberizadas. Uno de los cambios más notorios que cabe destacar en el análisis de los procesos de cambio que tienen lugar en nuestras ciudades es la emergencia de nuevos movimientos urbanos. Se constata, desde hace más de una década, una corriente soterrada de movilización y acción colectiva para la materialización de nuevas ideas de intervención local con una clara voluntad transformadora que ha de ser estudiada de forma integral. Más allá de Benkler y otros teóricos del cambio tecnológico que inciden en la hipótesis de la determinación que imprime la cultura digital en los actuales procesos de cambio (Benkler, 2015) (Blokland y Savage, 2008), todo análisis de las nuevas formas de mediación en el ámbito urbano pasa por considerar la dimensión subjetiva y las latencias que configuran la experiencia de transformación de las ciudades, en la medida que las subculturas que hoy rompen las normas sociales establecidas, así como los cambios que afectan a los modelos de representación y participación política local, trascienden toda lectura tecnocentrista.

Desde este punto de vista, el nuevo sensorium de la cultura pública que acompaña los procesos de movilización del activismo digital nos sitúa ante el reto de pensar las dinámicas culturales en las ciudades pensando en los sujetos y en la creciente circulación de información, que hace posible lo que Indovina denomina ciudad difusa (Indovina, 2012) y Richard Florida ciudades creativas (Florida, 2009), a partir de las contradictorias lógicas expansionistas de valorización con la consecuencia, inevitable a juzgar por la ley de hierro del desarrollo tecnológico, de la comodificación de las culturas e industrias locales. En este proceso, las NTIC proporcionan herramientas y espacios para construir dialógicamente un nuevo sentido de identidad y pertenencia, más allá de la participación en una comunidad o lugar de adscripción territorial. Las memorias digitales que circulan en la red dan lugar así a nuevas derivas urbanas y formas originales de ciudadanía con las que es necesario pensar de forma distinta el espacio público y la 
democracia. En buena medida porque los nodos virtuales son puntos nodales y espacios estratégicos generadores de nuevas dinámicas sociales, tanto como atractores de movilidad y apertura al cambio social en los procesos de desenvolvimiento en un tiempo interregno, un tiempo-encrucijada de transición entre formas jerarquizadas, informacionales, de representación, y nuevas dinámicas de cooperación social. En el presente artículo, se plantea una aproximación a la tecnopolítica como un proceso de constitución determinante de la nueva cultura urbana en función de la estructura de sentimiento de las multitudes conectadas que hace realidad el proceso de apropiación del espacio público desde una concepción praxeológica del derecho a la ciudad. Ello apunta a una dimensión propia del análisis institucional, al imbricar elementos contextuales internos y externos de referencia. Todo diagnóstico y análisis de los procesos de remediación del activismo digital pasa, desde este punto de vista, por una lectura integral del acontecimiento e interacción en las redes de acción colectiva. Pese a la relevancia de la comunicación de los nuevos movimientos sociales sigue prevaleciendo en la literatura académica los enfoques de movilización de recursos o la omisión, como en los años setenta y ochenta, del proceso de mediación como una suerte de caja negra relativa a la producción de identidades. Pero sabemos que la generación de comunidades virtuales, la proyección imaginaria de espacios autónomos de articulación y la generación de una institucionalidad y narrativa emergente, en parte gracias a la socialización de las tecnologías de la información y las lógicas tramadas de descentralización, apuntan en otro sentido.

El papel de la cultura digital en los nuevos movimientos urbanos ha sido disruptivo tanto en la dimensión política y la participación ciudadana, como en la narrativa y discursos, los modelos de organización y acción colectiva y los procesos de representación y expresión ciudadanas. En otras palabras, el ciberactivismo plantea no sólo un problema de método o meramente instrumental a la hora de abordar el estudio de estos nuevos fenómenos, sino esencialmente un dilema conceptual que justifica la pertinencia de definir las nuevas formas de intervención social considerando el derecho a la ciudad como un derecho a la comunicación y los derechos de ciudadanía en el mundo de las redes que nos tocan vivir, imaginar, hackear, y hasta impugnar, como espacio privilegiado de producción de lo común. La emergencia de los nuevos movimientos urbanos articula la voluntad de autonomía, la reconstrucción del derecho a decidir y la defensa del dominio público como proceso de transformación del medio social o entorno urbano en términos de lucha por el código (Formenti, 2017). El estudio del desarrollo local desde una mirada comunicacional es, en consecuencia, necesaria para comprender las redes de confianza y trabajo colaborativo reconociendo la dimensión performativa de todo intercambio e interacción social en red en los actuales procesos de cambio social. En esa línea, una concepción actualizada de análisis de la cuestión urbana exige un mayor interés por las políticas culturales, la promoción de identidades políticas con apoyo de procesos abiertos y dialógico de comunicación en el ámbito local, y una mayor conciencia 
teórica sobre el papel de la comunicación en procesos de transformación del territorio. Experiencias históricas como el 15M o Plaza Tahir ilustran la importancia de la mediación digital en la construcción de dinámicas autónomas de intervención. En otras palabras, la autonomía de acción de los nuevos movimientos urbanos no es fruto de una concesión ni impuesta por terceros, no se trata, en fin, de una concesión previa de lo apropiado ni solo un proceso determinado por la oportunidad y los recursos disponibles (Neuman, 2008). La capacidad de hacer nuestro del ciberactivismo en el ámbito local implica no sólo la tarea de ensamblar "sino la más arriesgada y fecunda de rediseñar los modelos para que quepa nuestra heterogénea realidad" (Martín-Barbero, 2002: 17). De esta manera, la herramienta tecnológica (que es tanto un espacio físico de encuentro como un recurso material) se transforma en un objeto relacional y de resignificación de las prácticas diarias de los sujetos involucrados, generando un proceso de re-codificación. En las siguientes páginas, vamos a tratar de definir el marco lógico de análisis de tales procesos definiendo las variables y dimensiones más determinantes, así como la discusión teórica que ha marcado el debate académico sobre la naturaleza de la oleada de protestas y las nuevas formas de acción colectiva.

\section{Mediación social, cultura urbana y cambio social. Estado del arte}

En la tradición de los estudios de comunicación y cambio social, el concepto de apropiación tecnológica ha sido históricamente central para ilustrar los procesos de adaptación creativa de las culturas populares ante las transformaciones técnicas tanto de la composición del trabajo como de la reproducción social. Esta es una categoría que piensa la mediación social como un proceso en movimiento, y la investigación en comunicación, parafraseando a Jesús lbáñez, como una práctica teórica que renuncia a la razón sedentaria para asumir la mirada nómada de las redes, espacios e intersticios de producción de lo común. En el propio proceso de apropiación, sabemos que la gente común desarrolla la capacidad creativa de nuevos usos y significados de los objetos y/o procesos apropiados como un proceso de innovación social en el software propietario, de las radios comunitarias a la televisión en la rua, del diseño gráfico de la agitprop al arte público y el graffiti. Realidades como los Smart Mobs y la autogestión de procesos de intervención colaborativa apuntan, en esta dirección, hacia una de las lecturas más productivas del diagnóstico sistematizado en el estudio de campo sobre la materia, partiendo de la idea de la importancia de visibilizar los modelos de democracia local a través de Internet que fortalecen el capital simbólico y material de las culturas populares en la apropiación de las nuevas tecnologías. Ahora, para definir un abordaje estructural y sociocrítico de tales procesos, es necesario distinguir a este respecto entre Capital Social (lo que Cees Hamelink (2000), denomina Capital Informacional) y Cultivo Social (la cultura, y desarrollo de redes ciudadanas articuladas en comunidades, más allá de las condiciones objetivas o materiales) (Vizer, 2003). Ambos componentes deben ser considerados en la extensión de las nuevas tecnologías para una ciudadanía activa, especialmente el Cultivo Social, por cuanto constituye la trama expresiva de 
formaciones de sentido en la vida cotidiana que permiten formas organizativas de calidad y complejidad superior, transformando como consecuencia a los actores sociales en agentes activos del cambio del entorno. Como bien ha criticado el profesor Navarro (2003), el discurso sobre el Capital Social trata por principio de remplazar el análisis del poder entre clases, razas y géneros, y sus consecuencias en términos de políticas públicas, por una lectura economicista e instrumental de las relaciones sociales (Sierra y Gravante, 2012). Es preciso, en este sentido, pasar de una lectura "capitalizada" de los procesos de cambio que introduce el activismo digital a una visión sociocrítica y estructural del proceso intersubjetivo de apropiación social de las nuevas tecnologías que recupere la potencia del habitus y la capacidad creativa de la experiencia de los sujetos y actores sociales. Desde este punto de vista, se puede hablar de diversos niveles de complejidad a la hora de analizar el proceso de apropiación de las TIC's por parte de los actores sociales: de la información a la deliberación, de los procesos de consulta y dinamización cultural a la elección y decisión vinculante, pasando por la propia participación en la política de redes y modernización de las llamadas ciudades digitales. La complejidad y posibilidades del uso y gestión de los sistemas avanzados ofrecen pues diferentes alternativas para el diseño de un entorno abierto de interacción tal y como se observa en el trabajo de campo sobre la materia (Sierra, 2019).

Considerando la revisión del estado del arte y en virtud de los puntos de anclaje del marco lógico de partida, en nuestro proyecto de I+D observamos cómo la ciberdemocracia impulsada por los nuevos movimientos urbanos sugiere un proceso de uso y apropiación de las nuevas tecnologías en procesos de cambio social según una estructura de código abierto, por lo que han de ser contempladas las identidades múltiples y difusas de lugares y pertenencias, así como la exigencia de mayor permeabilidad de la planificación y desarrollo de autoridades locales. De los estudios realizados en la materia, se observa que existe una correlación directa entre el grado de apertura y la mayor o menor diversidad de actores locales participantes en los procesos de apropiación social activa de las políticas de ciudadanía digital y la efectiva incorporación de estos nuevos medios y equipamientos culturales en proyectos de desarrollo comunitario. Dos factores determinantes de esta positiva relación son la definición o no de políticas públicas municipales con participación social de la ciudadanía y el grado de desarrollo del capital cultural compartido por la población en materia de comunicación, derechos culturales y capacidad de autoorganización en redes sociales más allá de las plataformas GAFAM. Bien es cierto que "Twitter, unido a redes sociales como Facebook, contribuyó a la generación de una auténtica revolución en la comunicación social; un fenómeno que Castells et al., (2006) ha denominado autocomunicación de masas, que le permitió a estos movimientos sociales, superar la limitación y la censura de los tradicionales medios masivos de comunicación y a la vez ayudó a su visibilizaciòn" (Gil y Guilleume, 2017: 136). Pero el activismo digital de los nuevos movimientos urbanos va más allá y ha de ser concebida como una remediación para el cambio social que irrumpe en la 
esfera pública mediante la difusión de un contenido relevante de proyección social con voluntad de propagación y articulación social, esto es, como una forma de acción política autónoma. "El poder que se ha conseguido con los Nuevos Movimientos Sociales de la mano del activismo ha logrado una transformación considerable en la comunicación y un nuevo resurgir en el aspecto político para la sociedad, consiguiendo un desprendimiento de los medios tradicionales que siempre se hacían presentes, arrebatándoles a los medios masivos de difusión la hegemonía informativa y narrativa, dando espacio a un activismo comunicativo que busca una mejor y mayor forma de expresión. Es así como el efecto de la comunicación inmediata y propagada dentro de una sociedad carente de acción, información y comunicación, reacciona ante la opción de cambio. El actuar de las protestas sociales se reduce inicialmente a un encuentro tras pantallas que culmina con su acercamiento presencial trabajado por un hecho antecedido de forma tecnológica" (Cortés y Garzón, 2017: 109).

Tomando en consideración las transformaciones experimentadas en el campo de la comunicación y la recomposición de las esferas públicas y privadas a nivel local, con la crisis del concepto de servicio público y la progresiva individualización y vaciamiento de los vínculos comunitarios, el trabajo de campo que venimos desarrollando constata, de hecho:

1. La multiplicación de foros de discusión y el desarrollo de espacios de expresión y visibilidad social de grupos de población tradicionalmente excluidos de los medios convencionales de información, en particular jóvenes, migrantes y mujeres.

2. La proliferación de grupos y colectivos sociales de movilización e intervención política en procesos de video y/o netactivismo a nivel local.

3. La extensión de redes globales de movilización basadas en experiencias participativas de creatividad social en el uso y apropiación de las nuevas tecnologías para el desarrollo social.

Ante la constatación de estas nuevas realidades emergentes que actualizan la cuestión urbana y el derecho a la ciudad, en esta ocasión desde una perspectiva comunicacional, cabe plantear, en consecuencia, numerosas las cuestiones a abordar desde la investigación. Entre otros, cabe plantearse: ¿Qué formas de agrupamiento social y extensión de las redes cívicas emergen con los sistemas telemáticos y desterritorializados de información y comunicación en las nuevas ciudades digitales? ¿Cómo son las formas y dinámicas de apropiación y uso social de las nuevas tecnologías que tienen lugar en los municipios que han planificado el uso público de estos recursos en el desarrollo local? ¿Qué inflexiones y cambios se están produciendo en las ciudades inmersas en proyectos de modernización tecnológica y socialización de la ciudadanía digital? ¿Qué desafíos y límites políticos y culturales plantean la introducción de los nuevos soportes mediáticos en el contexto de los planes de modernización de la Administración Local, desde 
el punto de vista de la construcción de la ciudadanía? ¿Qué subjetividad y cultura política comparten las multitudes inteligentes en línea? ¿Cómo son los procesos autónomos de participación y empoderamiento que tienen lugar en movilizaciones como el 15M? ¿A partir de qué lógicas se agrupan y organizan los nuevos movimientos sociales en red? ¿Qué factores y elementos distinguen las nuevas formas de acción colectiva de la ciudadanía digital y qué tipos de intervención producen en los procesos de desarrollo local más allá del eGobierno?

Sabemos que el ciberactivismo viene caracterizado por dos lógicas instituyentes: por un lado, por el código y la nueva arquitectura comunicacional de la ética hacker; y, por otra parte, por la dinámica comunitaria de intercambio y cooperación social en la que las políticas locales más han insistido. La constatación de esta ambivalencia, consustancial por otra parte a la dialéctica informativa, da lugar a una tensión o contradictoria dinámica entre la lógica de la gobernanza en la Administración Electrónica y la política de la comunicación de los sujetos y grupos activistas de la praxis distribuida en redes de cooperación. "Ciberespacio, gobierno y gobernanza comparten la misma partícula griega kyber, que devino en gober, y que tiene que ver con el hecho de dirigir, regir, guíar y originalmente se aplicaba a la navegación. Paradójicamente, con las ramificaciones de intereses se pensó que esa forma ciberespacial era ingobernable per se, lo que es etimológicamente un oxímoron, puesto que el ciberespacial es el espacio gobernado por antonomasia. Sin embargo, ese no es el problema actual, dado que hay cierto consenso en la regulabilidad del sustrato físico (servidores y cables) y sistemático (códigos y protocolos) de internet. La incógnita está en quiénes la gobiernan y cómo se gobierna" (Misseni, 2016: 264).

Como es conocido en la literatura académica sobre la materia, la filosofía de la Web 2.0 se basa en conceptos como simplicidad, compartición, generación de contenidos por parte del usuario y autorregulación, poniendo el valor fundamentalmente en la comunidad y en la información autónoma generada por los denominados prosumidores (Sierra y Gravante 2017). Aplicaciones como Wikipedia o Youtube son ejemplos de esta filosofía. En línea con esta idea, la incorporación del concepto de C2C al modelo y aplicaciones que se están desarrollando para las Entidades Locales, como resultado de los distintos programas de incentivos de la propia UE, apunta a un cambio de modelo institucional de la administración de proximidad, interpelado por la ciudadanía activa organizada autónomamente en los movimientos y redes informales de la sociedad civil. Del paradigma informacional a la mediación creativa de las singularidades proliferantes en la tecnocultura, se observa como resultado un cambio en las formas de participación y organización de la ciudadanía en lo que Sánchez identifica como lógicas latentes y organizaciones híbridas más propias de la subpolítica. "Este tipo de ciudadanía en stand-by, a la espera de que las instituciones asuman sus demandas y desarrollen canales para ello, describe un comportamiento pre-político en el cual discuten de política, consumen noticias 
relacionadas con este ámbito o hablan de temas sociales. Lejos de ser una ciudadanía al margen de los procesos de decisión muestra una mayor propensión a entablar conversaciones políticas que otros individuos asociados a organizaciones, mostrando altos niveles de confianza social con el entorno y confianza política. Un modo de hacer política con un menor grado de institucionalización fuerza (en esta dirección) a ir más allá de las instituciones representativas del sistema político (subpolitica) a veces sumergida en el ámbito de experiencias subjetivas y la vida cotidiana" (Sánchez, 2016: 34).

Considerando esta hipótesis de partida, es preciso por ello explorar, más allá de los abordajes neoinstitucionalistas de la ciencia política, un análisis sociocultural de:

- Las tipologías y experiencias creativas de participación ciudadana en el entorno digital.

- Los actores y procesos de articulación de tejido social y redes cívicas a través de entornos virtuales.

- Las metodologías y tipos de intervención de las organizaciones y movimientos sociales en procesos de videoactivismo y movilización en red.

- Los nuevos imaginarios urbanos y formas de apropiación de la cultura digital en infraestructuras públicas o procesos informales.

- Las técnicas y usos sociales de los medios y dispositivos de intervención política y social de la ciudadanía en prácticas de activismo digital.

- Las estéticas emergentes de las nuevas formas de producción de lo público y procomún.

Se trata en suma, lo que es poco habitual en la investigación, de definir e identificar con precisión las principales líneas de desarrollo democrático de la Sociedad de la Información en el ámbito local, analizando tanto las formas de autonomía y apropiación social de las nuevas tecnologías como la articulación de procesos de cambio social de los actores locales, incluyendo las dimensiones institucionales tanto como las redes no formales de expresión de la ciudadanía en sus mundos de vida en lo que podemos definir como redes críticas de empoderamiento social. Esta perspectiva difiere de la visión habitual en los estudios especializados en la materia que tienden a poner mayor énfasis en las dinámicas instituidas de gobierno a la hora de abordar los procesos de cambio e innovación tecnológica en el ámbito local. 
Desde los años setenta, el paradigma "clásico" de evaluación del sector público, que tiene su origen en los procesos de reforma y modernización del gobierno de los Estados Unidos, viene basando sus estrategias de conocimiento y diagnóstico de las políticas locales y, en general, de la Administración Pública en métodos fuertemente positivistas, por el que la noción de interés público, a priori, definida por los "expertos" o la clase política. En línea con este planteamiento teóricometodológico, una década después, en los años ochenta y, por supuesto, en los noventa, en virtud del discurso y la filosofía pública neoliberal, se impone el llamado New Public Management, que asume los métodos y criterios de evaluación de la empresa privada, habitualmente cuestionables para la medición y evaluación de las políticas públicas. Desde finales de los noventa se viene así desarrollando una lógica de investigación y práctica institucional basada en el concepto de Networked Governance y de "valor público", que prioriza fuertemente el papel de los ciudadanos, de su participación y compromiso con las políticas públicas en calidad de usuarios y consumidores de servicios vaciando de contenido sustancial su rol en la planeación estratégica del desarrollo local. La aproximación cualitativa en los estudios de caso y experiencias de gobernabilidad e impacto de las políticas locales debe, en buena medida, su extensión a esta lógica de análisis de las motivaciones y nivel de satisfacción de los destinatarios de las políticas públicas.

De acuerdo a esa filosofía y lógica del método, el eGovernment Economics Project (eGEP) fijó, en el ámbito de la UE, las bases de lo que debía ser la matriz conceptual de medición de resultados del e-gobierno partiendo de la base que cualquier institución o programa público debía perseguir tres grandes objetivos principales: eficiencia, atención centralizada en el usuario y transparencia, principios en virtud de los que se desarrollaron alrededor de noventa indicadores para evaluar los diferentes subcampos derivados de estas tres metas prioritarias. Desde el mundo académico han sido muchas las críticas expresadas al llamado "supply side benchmarking" con las que se implementaron las metodologías de diseño y evaluación de la ciudadanía digital. No viene al caso en este artículo ahondar en esta materia ya tratada en otros trabajos pero sí al menos apuntar, en la revisión del estado del arte, una lógica de conceptualización que, como en el caso de la Agenda de Lisboa, por más que abunda en la idea de creatividad, democracia y autonomía termina por cercenar toda posibilidad de transformación que, en parte, explica la crisis de legitimidad de la propia UE y los poderes locales (Sierra, 2010a) (Sierra, 2010b), (Sierra, 2013).

Los estudios sobre ciberdemocracia vienen no obstante replicando esta visión dominante al repensar la revolución digital en el análisis de la cuestión urbana. La política de implementación del e-gobierno según los patrones del SIBIS (Statistical Indicators Benchmarking the Information Society), financiado por la Comisión Europea (IST-2000-26276) entre enero de 2001 y septiembre de 2003 (Sexto Programa Marco), permean de hecho los discursos científicos e institucionales 
sobre el desarrollo innovador de la Sociedad de la Información de las ciudades, limitando la teoría y práctica de las ciudades digitales a nueve cuestiones básicas:

1. Acceso a las telecomunicaciones.

2. Investigación y desarrollo de Internet.

3. Seguridad y confiabilidad de los sistemas de información.

4. Educación.

5. Empleo.

6. Inclusión social.

7. Comercio electrónico

8. Salud.

9. Y administración electrónica.

Es posible documentar no obstante otras estrategias y criterios metodológicos más integradores y coherentes para la comprensión y diagnóstico de los procesos de cambio local ligados al activismo digital y a la emergencia de un nuevo tipo de subjetividad política y de ciudadanía que demanda otra forma y dinámica de mediación social en la nueva cultura urbana.

\section{Metodología y diseño de investigación}

La extensión tecnológica de recursos y repertorios de acción de los nuevos movimientos urbanos redefine hoy la forma de producción y expresión de la ciudadanía. La consecución de una mayor potencia organizativa, la disposición autónoma de códigos culturales y la capacidad de intervención inmediata en lo local son factores que explican en parte un cambio de patrón en la dinámica de los municipios que están en el origen de la exigencia de nuevos modelos de gobernanza. Pero, como venimos señalando, la construcción de instituciones abiertas a la autoorganización de los movimientos sociales y la paulatina asunción de una cultura de servicio de la promoción y el desarrollo local participada son aspectos interdependientes que fijan, a modo de condiciones previas, las posibilidades de una nueva autonomía en la que la cultura digital es una herramienta fundamental de diagnóstico y propuesta de gestión que depende de otras variables ajenas a la estructura institucional de la administración. Por ello, nuestro análisis de la mediación social del ciberactivismo, a diferencia del diseño teórico-metodológico dominante en la literatura especializada en la materia, parte de un modelo no parametral de comprensión de los procesos de remediación más que de la sistematización y evaluación de resultados. Pues, en el estudio del activismo digital, interesa sobremanera las configuraciones sociopolíticas y el imaginario de los actores involucrados a fin de comprender los procesos de mediación social en las formas emergentes de ciudadanía. Por ello, el diseño de investigación, además de las etnografías, grupos de discusión y entrevistas a profundidad, propone metodologías participativas como el diseño de laboratorios ciudadanos centrados en las demandas, visiones y discursos de los actores locales. Así, el desarrollo de la aplicación interactiva resultante de las conclusiones de la investigación social permitirá describir los repertorios de acción, las 
narrativas y formas de organización emergentes y su despliegue en los procesos de transformación del territorio o de politización de la cuestión urbana del ciberactivismo, desde una perspectiva holística e integral.

El estudio del capital informacional y de los procesos colectivos de apropiación de las nuevas tecnologías digitales a través del análisis de experiencias y del impacto en el desarrollo local puede contribuir, en este sentido, de manera decisiva, a abrir la agenda de investigación sobre la nueva cultura urbana en la producción del espacio público problematizando radicalmente el desarrollo de los sistemas y servicios avanzados de comunicación digital en el proceso de modernización de la Administración Local, incorporando los desbordamientos, dinámicas de autogestión y posibilidades creativas de las prácticas de empoderamiento de los nuevos movimientos urbanos como actores políticos centrales en el proceso de transformación y planificación del territorio. Desde este punto de vista, pensar la ciudadanía digital o las formas de participación local en red de la población en las llamadas ciudades creativas va más allá de:

- Eliminar la brecha digital que afecta a determinados sectores desfavorecidos de la sociedad e incrementar el porcentaje de población que usa las TIC.

- Ofrecer espacios de comunicación directa entre la ciudadanía y las instituciones que permitan actuar en e-Democracia.

- E incorporar la cultura y los saberes locales al banco de conocimientos global de Internet, que garantiza su perduración en el tiempo y su difusión en el espacio.

El rol de las redes críticas cívicas en el gobierno local apunta en este sentido a repensar la cuestión urbana como un ámbito de investigación por explorar, tanto empírica como conceptualmente, desde otras matrices epistémicas más socioanalíticas, tal y como hemos argumentado en la introducción. Atendiendo a la multidiplinariedad y transversalidad deseables en el complejo mundo de la cultura digital, más aún considerando que estamos ante procesos de acción y movilización colectiva, nuestro proyecto de investigación plantea, en lógica coherencia, un enfoque sociopráxico de integración topológica y de pluralismo teórico-metodológico, para una aproximación exploratoria de un ámbito apenas tratado más allá del tecnodeterminismo o el pancomunicacionismo idealista. Entendiendo la transversalidad como "instrumento organizativo que pretende desarrollar estrategias, herramientas e instrumentos que, dentro de la estructura organizativa sectorial, permitan adaptarse mejor a las exigencias de una realidad muy compleja" , el diseño de investigación, con una marcada matriz comunicacional, integra diversos aspectos económicos, sociales, culturales y políticos, además de los específicamente tecnológicos, acorde con la necesaria multidisciplinariedad de análisis del fenómeno objeto de estudio. Si pensamos en 
un concepto transversal, complejo o multidimensional como el de ciudadanía (abordable desde la Ciencia Política y la Comunicación, pero también desde el Derecho, la Antropología o la Historia) es evidente tal afirmación. Por poner el caso hoy de los estudios urbanos de las ciudades digitales, observamos, en los escasos análisis aplicados en España referencias de la geografía crítica, de la economía aplicada, de la investigación en políticas públicas, análisis de ingeniería de telecomunicaciones, de políticas culturales, de comunicación y usos sociales, hasta de antropología visual y de la cultura, por lo general de forma dispersa y desagregada desde el punto de vista del análisis de variables. En nuestro estudio, procuramos por ello un diseño de investigación que trate de explorar una muestra significativa de experiencias locales, tanto en la UE (Italia, Portugal, España) como en América Latina (Brasil, México, Chile) a partir de estudios de caso que pueden contribuir a una formulación metodológica útil sobre la formación de las redes comunitarias con sistemas digitales de información y comunicación, combinando la diversidad de factores de la complejidad constitutiva de los procesos de activismo digital con un diseño dinámico y plural del marco teórico conceptual y un enfoque transversal y pluridisciplinario incluso en la propia composición del grupo de observadores: conceptualmente, al abordar el análisis de sujetos, redes, infraestructuras y espacios; y, disciplinariamente, al incorporar comunicólogos, politólogos, sociólogos, ingenieros e investigadores de estética, antropología y estudios visuales. Esta lógica del método puede ser complementado con propuestas como la Métrica v3 de sistematización de las actividades que dan soporte al ciclo de vida del software en procesos de egobierno para proporcionar o definir sistemas de información que ayuden a conseguir los fines de toda organización mediante la definición de un marco estratégico para el desarrollo de los mismos. Pero no es el objeto del presente estudio, tal y como hemos detallado. La fundamentación metodológica de la investigación se inspira más bien en la tradición de la Comunicación Participativa para el Cambio Social que, desde la educación popular, y la tradición de los estudios latinoamericanos de comunicación para el desarrollo local vienen implementándose a uno y otro lado del Atlántico. Los aportes en esta línea permiten, en el análisis del activismo digital y el estudio de los nuevos movimientos de protesta en las ciudades, identificar los actores, implicados y las diferentes posiciones o "lecturas" percibidas por los sujetos políticos de una determinada comunidad, generar conocimiento sobre las formas de innovación social de los actores colectivos, visualizar los discursos e imaginarios urbanos de los nuevos actores locales inmersos en procesos de movilización colectiva y sistematizar la tipología de repertorios de acción y códigos culturales puestos a disposición en la lucha por el código y el ejercicio del derecho a la ciudad.

En definitiva, la propuesta teórico-metodológica procura de este modo construir conocimiento en común, tal y como lo viven o perciben los sujetos, comparando datos cuantitativos y cualitativos con las opiniones y experiencias manifestadas por la población local desde los mundos de vida. Solo así es posible contrastar y analizar la densidad gruesa de constitución de las nuevas plataformas de 
intercambio de la ciudadanía poniendo en valor tanto su capital intelectual y social como el propio proceso de movilización para el cambio y empoderamiento de recursos como las nuevas tecnologías digitales, desde el punto de vista del desarrollo sociocultural.

\section{Análisis}

A partir del análisis empírico de diferentes experiencias de activismo digital y del proceso de apropiación social que han contribuido a revitalizar el gobierno y desarrollo local, es posible observar varias tipologías, factores y discursos que acompañan al cambio social. Todas ellas coinciden en territorializar, desde lo concreto, el proceso de mediación y articular el plano físico y virtual de intervención a lo largo de todo el proceso de movilización. En tanto que proceso de transformación articulador de organización y activismo social, el ciberactivismo se distingue por:

- La elevación del nivel de conciencia.

- La contrainformación.

- El despliegue de diversas formas de autoreconocimiento

- La organización en red

- El desarrollo de espacios autónomos de resistencia.

- Y la continua apelación a la movilización colectiva.

Como comunicación transformadora con voluntad emancipatoria, la acción conectiva que promueven los nuevos movimientos urbanos procura por lo mismo en todo momento:

- La participación política como irrupción.

- La construcción de un nosotros que desborda categorías modernas de identificación.

- El despliegue de las emociones y la vinculación social (Reguillo, 2017: 100).

Considerando lo antes expuesto en la muestra objeto de análisis, y a tenor de otros casos de estudio y el análisis comparado, se observa que, en las experiencias locales de activismo digital, tiene lugar, las siguientes pautas y procesos de socialización:

- En general, los estudios de caso analizados constatan un bajo nivel de trabajo colaborativo pese a la clara y decidida voluntad de participación y democracia directa en los espacios delimitados en la red. De ahí que, a tenor de los resultados cuantitativos y cualitativos testados, se concluya que el nivel de participación y apropiación social de la cultura digital resulte más bien un discurso e imaginario que la práctica real y concreta. 
- La mayor dificultad objetiva observada con relación al uso y apropiación de las nuevas tecnologías en procesos de ámbito local es su traducción en políticas de desarrollo endógeno integradas, de acuerdo a una visión transversal que considere los objetivos estratégicos de articulación social del derecho a la ciudad.

- Existe, por lo general, un déficit de información y saber relativo a la realidad local y a las posibilidades de aplicación de las nuevas tecnologías, más allá de vindicaciones de justicia global, debido a la ausencia de sistematización de experiencias locales anteriores sobre la cuestión urbana.

- Se observa, en consecuencia, como principal conclusión, a este respecto, una brecha cognitiva entre discursos normativos difusionistas del desarrollo local de las autoridades del gobierno de proximidad de turno y la acción política efectiva de los nuevos movimientos urbanos, en el marco de notorias carencias estructurales y agenciales, conforme a la racionalidad técnica y apolítica del proceso de innovación, que se constata en los discursos registrados por los actores locales entrevistados.

- Del análisis de los estudios de caso, se concluye, en fin, que las experiencias de empoderamiento y participación de la ciudadanía a través de las redes sociales son todavía insuficientes, cuantitativa y cualitativamente desde el punto de vista de la cuestión urbana y el alcance de las transformaciones vindicativas en el marco del proceso de reproducción local. Los usos dominantes, los escasos recursos materiales y humanos dispuestos a tal fin y, sobre todo, la limitada conciencia de las alternativas de repertorios de acción que considere el potencial liberador de las nuevas tecnologías informativas en los procesos de desarrollo local no han permitido aún el despliegue de prácticas innovadoras capaces de transformar significativamente las formas de gobierno y construcción de lo común.

- El dominio de una concepción pragmática en los procesos de mediación de los nuevos movimientos urbanos termina, como resultado, colonizando usos liberadores y creativos de construcción de una nueva economía moral de la multitud inteligente en red, afectando los modelos y lógicas de diseño de la intervención social, y los patrones y criterios de planeación y evaluación de las políticas públicas, tal y como se observa en la mayoría de casos analizados.

El reto, en fin, es tratar de definir la participación como apuesta por una democracia radical y pluralista, trascendiendo la definición de la cultura como recurso que imprimen las políticas internacionales de desarrollo en la gestión, almacenamiento, distribución y organización del acceso a los bienes simbólicos 
según las condiciones de circulación y valorización transnacionales del capitalismo, comenzando por replantear las directrices y prácticas instrumentales de intervención de los nuevos movimientos sociales. En la medida que la ciberdemocracia proyecta un nuevo escenario, o espacio público, nuevos métodos y posibilidades democráticas para la participación activa de la ciudadanía, y sobre todo una nueva concepción del espacio y de la mediación con el concurso activo de la población, esta exigencia se antoja prioritaria en nuestro análisis a la hora de redefinir las formas de reproducción social así como los roles y el protagonismo de los actores locales, cuestionando la noción misma de ciudadanía y el marco jurídico de la participación en el Estado en el marco de evolución del Estado-nación al Estado móvil que prefigura el Capitalismo Cognitivo. En otras palabras, pensar hoy la participación ciudadana debería significar, localmente, reflexionar sobre las mediaciones y las distancias, las prácticas culturales y los marcos cognitivos de reflexividad e imaginación política en los límites de la institucionalidad considerando la dimensión instituyente que imprimen estos nuevos movimientos urbanos con el ciberactivismo.

Una de las características más destacadas del actual proceso de globalización cultural es, sobremanera, la articulación de un modelo de regulación institucional, basado en la explotación intensiva del espacio y, complementariamente, la dispersión de las actividades económicas de forma descentralizada, junto con la concentración de actividades terciarias de alta remuneración como las industrias de ocio y consumo cultural en polos territoriales o las llamadas ciudades creativas, tal y como hemos visto. Esta lógica de la mediación reestructura el desarrollo territorial imponiendo una distribución urbana y unas necesidades de gestión y organización de la economía de la comunicación y la cultura, en función de las exigencias de valorización del capital. Como consecuencia de esta lógica o inercia en las políticas públicas, tienen lugar, a nivel subestatal, procesos de remediación alternativos. Si el poder habita el sentido y el sentido es fuente del poder, como sentencia Escobar, pensar las políticas culturales y, más allá aún, la forma-Estado y el devenir del capitalismo, pasa justamente por deconstruir el régimen discursivo de las políticas de base local en la globalización, problematizando la cuestión epistemológica de los lugares y el poder de la religancia. Por ello, hoy más que nunca es preciso formular un enfoque enactivo, ecológico y sociocrítico, desde la inmanencia de la nueva biopolítica, de las prácticas comunicativas y las políticas de representación y reconocimiento que cercan y atraviesan el conjunto social del vivir y soñar de los sectores populares, tal y como se vislumbra en nuestro proyecto. Pues toda ciudad es, antes que nada, un constructo fruto de la memoria cultural, de la historia económica y la política de la vida cotidiana producida, parafraseando a Walter Benjamin, por los soportes físicos de configuración de los pasajes, flujos, intercambios y condiciones materiales concretas de los sujetos sociales en función de la necesidad de producción de la vida en común. En otras palabras, la ciudad, antes que nada, es artificio, el arte de un hábito cultural, de un habitus y humus producido en común como estilo o medio ambiente sociocultural, organizado performativamente por intercambios y lógicas de 
enunciación definidos a partir de las ecologías de vida, desde el plano de la inmanencia, lo que exige repensar el espacio y su engarce con lo simbólico como punto neurálgico de los problemas contemporáneos del postdesarrollo.

La descentralización, la defensa del territorio, ha sido siempre, sin duda, como el derecho de autodeterminación, un problema político estratégico. Y con él el problema de la participación ciudadana y la autonomía en las formas modernas de gubernamentalidad que hoy, de nuevo, vuelve a actualizar las discusiones sobre el derecho a la ciudad con la emergencia de lo que David Harvey denomina ciudades rebeldes. Hablamos de geografía, de ordenación del territorio, pero también, como es lógico, de cultura, del poder de decir y ordenar. De ahí, como hemos tratado de mostrar, la pertinencia de enfoques postestructuralistas y transversales, como marco lógico de interpretación de la revolución digital. Pues la cultura como problema comprende, como advirtiera Williams, el conjunto total de la vida social.

Si el espacio local es un ámbito territorial de lo próximo y cercano, de lo doméstico y familiar, el espacio, en fin, de convivencia y sociabilidad básica que, por lo mismo, remite a lo abarcable y apropiable públicamente, a nivel individual y colectivo, repensar hoy en las ciudades el ciberactivismo como fenómeno de ciudadanización constituye un problema de Estado que, más allá de las imágenes e imaginarios urbanos de progreso y del acceso a recursos culturales como internet en términos de democratización cultural, interpela los dispositivos de poder y reproducción del capital desde los mundos de vida y la producción mancomunada de cooperación y apropiación del capital simbólico y los territorios in/habitables por las clases subalternas. Pero tal exigencia es excepcional, paradójicamente, pese a su centralidad, en nuestro país y, en general, en las ciencias sociales, en un contexto, además, marcadamente adverso, en plena hegemonía y apogeo del discurso modernizador del neodifusionismo liberalconservador que comenzaría por identificar los problemas de representación y reconocimiento, el problema de la identidad y la diferencia, en la nueva cultura urbana, en función de la lógica de la Cultura como Recurso tal y como observamos en la mayoría de análisis sobre los nuevos movimientos urbanos. En términos de Jameson, el espacio urbano se termina así convirtiendo, por imposición de un renovado discurso del inconsciente ideológico, en ámbito privilegiado de valorización del Capital en tanto que lugar natural de intercambio material y simbólico. Pues, desde el punto de vista genealógico, la modernidad es un relato que depende de la mediación social, del proceso de articulación de lo físico y lo simbólico. La colonización del territorio por medio de las fantasías electrónicas con las que habitamos el mundo y se teje, oníricamente, las formas de sujeción y dominio hegemónicos es, de hecho, el proceso estratégico determinante a partir del cual es posible explicar cómo hoy la geografía urbana puede ser objeto de múltiples alteraciones de acuerdo a las exigencias del capital rentista y los circuitos financieros internacionales en su lógica de flujos y movilización continua. Por ello, la pertinencia de una lectura sociocrítica, histórica 
y socialmente situada, y políticamente reflexiva, como la que aquí se presenta, es imprescindible en medio del escenario proliferante de análisis neoinstitucionalistas y positivos que impera en los colegios invisibles de las Ciencias Políticas y los Estudios Culturales.

Para quienes pensamos que no es posible traducir y comprender integralmente los problemas de las políticas públicas en comunicación y cultura sin una fuerte articulación lógica con las prácticas comunicativas, sin un compromiso intelectual y moral en el campo de la praxis, con las culturas populares y las multitudes proliferantes, todo análisis de los modelos de intervención social al margen de la dinámica de apropiación ciudadana del cambio social, de las luchas de los movimientos sociales por el código, sean las casas de cultura, las caracolas zapatistas de resistencia, o los centros sociales del movimiento okupa en Europa, se nos antoja improductivo o directamente cómplice con la reproducción de la colonialidad del saber-poder, particularmente redundante en la era de la subsunción de la práctica especializada del conocimiento que impone el Capitalismo Cognitivo. Pues la producción del sentido y la performatividad de los planes de desarrollo y ordenación del territorio, junto con las políticas culturales, son la base de mediación de las formas de reacomodo de la hegemonía. Esto es, lo decible y lo pensable son operaciones cognitivas que están articuladas con el proceso de reproducción de los regímenes de significación entre lo instituido y lo instituyente, que hoy implosionan cuando ni en el campo ni en la ciudad es posible vivir y habitar juntos con dignidad.

Desde este punto de vista, la lectura del fenómeno de la llamada ciberdemocracia admite en esta línea una fuga o deconstrucción socioanalítica de la plusvalía semántica de la política cultural, de lo deseado y no realizado, de lo sugerido y desplazado, de lo silente y sistemáticamente negado, una lectura, en fin, de las ausencias y las potentes formas populares de emergencia que contribuya de forma efectiva a la posibilidad de explorar nuevos espacios de esperanza para la idea, siempre figurada, de, en el sentido de Castoriadis, la autonomía frente al progrom neoliberal contra los sectores subalternos.

Como recuerda Arturo Escobar, la política de la emergencia muestra que puede haber, que siempre acontece con la multitud, la disposición en los lugares de formas comunales de inteligencia colectiva, que a la seguridad democrática sucede la potente articulación de formas creativas de autoorganización social en red. Sabemos que la colonialidad global del poder determina una distribución o lógica mundo-favela que, como resultado, problematiza la movilidad urbana e impone marcos restrictivos para la apropiación de los bienes comunes, para la circulación en los espacios urbanos. Esta y no otra lógica es la que configura la historia de la ciudad, el origen, en definitiva, del proceso universal de higienización de las culturas populares que da lugar hoy a los nuevos frentes de lucha y conflicto por el pase libre en Río de Janeiro o la conservación del patrimonio de proximidad de insignes teatros o cafés en Buenos Aires. 
Igualmente, sabemos que una de las principales consecuencias de la modernidad es la alteración de la experiencia del sujeto moderno en el tiempo y en el espacio, de acuerdo con la idea de desanclaje (Anthony Giddens dixit), haciendo del viaje, y en general, de la cinética, del impulso a la movilidad permanente, el nuevo espíritu del capitalismo, la razón de ser de la vida urbana. Y con ello un problema de definición de la vida social. Pues la ciudad no es un lugar, como la movilidad no es una transición entre espacios, sino sobre todo una relación con el territorio y con los otros. Y por ello un problema de política cultural, un conflicto vital del derecho a la ciudad, tal y como vemos en las luchas de Río, Madrid o Estambul. Pues, como se puede colegir, pensar el activismo digital en el ámbito subestatal es problematizar las ecologías de vida, el modelo de articulación de los vínculos en virtud de la demanda de nuevas formas de mediación y reconocimiento de las prácticas comunicativas autónomas.

Si el capital desconecta, aísla, desterritorializa, segmenta, externaliza y descompone las formas de religancia, una política democrática en materia de ciudadanía digital ha de comenzar por repensar estas lógicas. Pues el futuro de la cultura, y de la ciudadanía, pasa básicamente por trazar vínculos, estructurar espacios de cooperación, aprender, en fin, que compartir es crear, que politizar los lugares es imaginar rearticulaciones en común, hoy más que nunca posibles y necesarias. La idea de las redes críticas de empoderamiento, el sentido de las políticas culturales de base local descansa hoy, por ello, en lo que hemos convenido llamar el lenguaje de los vínculos. El empoderamiento de una comunidad local presupone, en este sentido, la capacidad para ponerla en relación con otras comunidades. La participación y autonomía es, por tanto, descentralización y recentramiento comunitario, pero también apertura y ligazón. Sea en los ámbitos de proximidad, construyendo ecologías políticas regionales (caso del Pacífico colombiano estudiado por Escobar) o redes interurbanas, como en el 15M en España, sean movimientos alterglobalizadores como el Foro Social Mundial, o iniciativas intergeneracionales como el voluntariado digital en Andalucía. En todos y cada uno de estos casos e iniciativas sociales, existe la voluntad común de proyectar una dialéctica de la dialogía en la producción de las políticas culturales, una dinámica social creativa que necesariamente determine todo agenciamiento, a partir de las tensionalidades entre proceso social e institución, como ilustran los autores ante el problema de sostenibilidad del objeto de estudio. Solo así es posible construir nuevos universos posibles.

\section{Discusión y conclusiones}

Las nuevas prácticas de activismo digital involucran nuevos lenguajes, saberes liberadores, formas organizativas singulares, procesos autónomos de intervención que reconfiguran el espacio público y la propia política. Ello demanda de la investigación social análisis sobre:

- El discurso y la mediación social. 
- La estética y códigos culturales de reconocimiento de los movimientos sociales.

- La consideración de las formas autónomas de organización de las comunidades en la definición de lo público.

- La producción de los imaginarios que están en el origen de las nuevas prácticas políticas y de intercambio.

Ahora, la relación entre movimientos sociales y procesos de comunicación es, en el ciberativismo, compleja y exige un abordaje multidimensional, más allá de la racionalidad instrumental o el idealismo pancomunicacionista. La transversalidad, el enfoque pluridisciplinario y ecológico de nuestro objeto de estudio, viene además justificada por la perspectiva teórica de la que es deudora. Cuando menos, a nuestro juicio, "pensar lo común desde esta perspectiva implica considerar la centralidad que tienen las prácticas sociales orientadas a garantizar y cuidar aquello que se comparte. A igual que el capital, lo común tampoco es fijo, estable o dado de antemano, al contrario, hay tensiones, conflictos y relaciones de lucha entre su constitución y existencia. Lo anterior significa que lo común solo es posible a partir de relaciones sociales que - estando atravesadas en mayor o menor medida por el capital - luchan por su constante recreación o en términos de John Holloway por su constitución" (Modonesi, 2015: 54). Por otra parte, además, "Internet es una forma-contenido donde los procesos de transformación de una totalidad experimentan modificaciones en su estructura a causa de la dinámica social, el impacto de sus acciones y de las configuraciones materiales su cuerpo físico - y territoriales y de la división del trabajo. Internet, como espacio cibernético, es un generador de símbolos, sentidos y significaciones condicionadas por el tiempo. Este espacio es soporte de flujos horizontales que impulsan lo común, lo compartido o cooperativo, pero también de flujos verticales que nutren las tendencias utilitaristas o individualistas. En este punto, el análisis del poder y del dominio adquiere una centralidad difícilmente cuestionable" (Rodríguez y Martínez, 2016: 254). Cuando menos todo proceso de articulación entre cambio social y cambio comunicativo comprende tres campos y/o dimensiones: la organizativa, la subjetiva y la económico-política. "En la dimensión político-organizativa, la mediación comunicativa se ejerce a través de las dinámicas de articulación y la configuración de formas organizativas. En la dimensión simbólico-emocional, la mediación se expresa en la visibilidad, en la construcción de sentidos compartidos y en la movilización emocional. En la dimensión económico-productiva, la mediación se manifiesta en la configuración de un nuevo sentido de colectividad en la producción" (Bacallao-Pino, 2016: 65). En otras palabras, el ciberactivismo puede ser considerado una forma de intervención comprometida con el cambio social considerando actividades técnicas e instrumentales de alteración de los flujos de información, como la organización de procesos sociales por la autonomía de producción de sus representaciones y formas autónomas y antagonistas de articulación. La capacidad 
de configuración de espacios de interacción y comunicación interpersonal como contraesfera pública, en el sentido de Fraser, viene dada por la autonomía del desarrollo off y online de los actores locales. El capital político y social acumulado es lo que permite en este sentido la articulación de procesos de autodeterminación desde la lógica microblogging. Ello significa siempre una forma de mediación tecnopolítica que comprende tanto procesos de actuación mediática o simbólica en general como física.

\begin{abstract}
"Se trata en definitiva de procesos sociales diferenciados vinculados en complejas redes informales que promueven el cambio social o se oponen a la corriente dominante, síntomas de la violencia generada por el capitalismo neoliberal, señalan la crisis global, las lógicas depredadoras de esta y su incidencia institucional sobre el proyecto inconcluso del Estado del Bienestar. Comparten vínculos con otros actores no necesariamente idénticos pero sí compatibles, mejor hablar de compatibilidades que de identidad, en una movilización colectiva más amplia, como proceso de reconocimiento mutuo en la diferencia y la heterogeneidad, en el intercambio y la comunicación - como acción en común de experiencias contra-hegemónicas fuertemente arraigada en el uso estratégico, obviamente también como expresión identitaria, de los lenguajes y las herramientas que proporcionan las $\mathrm{TICs}$, sin líderes reconocibles, son inapropiables, asamblearios y horizontales, siendo muchas de sus estrategias comunicativas de carácter lúdico, carnavalizadoras y deconstructoras a través del humor y la utilización de significantes festivos" (Sabariego, 2017: 8).
\end{abstract}

Una nueva praxiología, de acuerdo con Padilla (2012), que constata cómo la red está siendo cuando se hace y mientras se piensa da lugar a una nueva dialéctica de lo público y lo privado pues, como medio Internet, es tanto contexto como acontecimiento, a la vez lo que habla y de lo que se habla como campo de disputa del sentido. De acuerdo con Reguillo, "quizás lo más relevante en la relación entre el acontecimiento (la revuelta), el lugar (la plaza, la calle, ciertos espacios) y la performance (el despliegue de una episteme, un modo de transmisión, una realización y un medio de intervenir en el mundo) es tender un puente entre el tiempo extraordinario de la protesta y el tiempo ordinario de la vida cotidiana, entre lo estructural y lo coyuntural, que encuentra en el emplazamiento las condiciones para abrir una política del estar - ser - decir juntos y juntas de otro modo (...) El emplazamiento concilia eso que llamamos lo privado - esa experiencia de dolor, angustia y hartazgo individual - con lo que ya no llamamos lo público, sino lo común" (Reguillo, 2017: 76). Los nuevos frentes culturales son hoy espacios porosos y fluidos, móviles, entre culturas y clases diferentes, así como espacios de lucha y confrontación por el capital simbólico y económico que exige un enfoque vertical tanto como lateral de la realidad, tal y como apunta el profesor González (González, 2012). La pertinencia de este abordaje viene dado por la centralidad de los nuevos movimientos urbanos en diversas latitudes y culturas constatando en todos los casos un proceso de remediación que justifican algunas tesis aquí expuestas sobre la lógica de flujos y la transformación de la comunicación para el cambio social, empezando por la 
propia crisis de legitimidad política como crisis de representación que afecta sobremanera a la autonomía y a la función vicaria de la mediación de actos sociales del espacio público local.

El futuro de nuestras ciudades depende, en este sentido, hoy más que nunca, de la capacidad que tengamos para articular una democracia de alta intensidad a partir de un proceso innovador de aprendizaje y cambio social. Es de desear que, en este sentido, las lecturas e hipótesis aquí apuntadas contribuyan cuando menos a alimentar la voluntad por conocer y explorar las nuevas luchas por el código y la ciudadanía en el nuevo período histórico que viven nuestras ciudades en la era digital.

\section{Financiamiento}

Este artículo es producto del proyecto de Investigación titulado "Ciberactivismo, Ciudadanía Digital y Nuevos Movimientos Urbanos" (CiberMov), referencia CSO2016-78386-P, financiado por el Programa Estatal de Fomento de la Investigación Científica y Técnica de Excelencia, Subprograma Estatal de Generación de Conocimiento del Ministerio de Economía, Industria y Competitividad y coordinado por el Grupo Interdisciplinario de Estudios en Comunicación, Política y Cambio Social (COMPOLÍTICAS) de la Universidad de Sevilla. 


\section{Referencias bibliográficas}

BACALLAO-PINO, L. (2016): "Dimensiones de la mediación comunicativa en los movimientos sociales latinoamericanos", Perspectivas de la Comunicación, Vol. 9 número 1, UFRO, Temuco, pp. 65-81.

BENKLER, Y. (2015). La Riqueza de las Redes. Cómo la producción social transforma los mercados y la libertad. Barcelona, Icaria Editorial.

BLOKLAND, T. y SAVAGE, M. (2008). Social Capital and Networked Urbanism, London, Blackwell.

CASTELLS, M. (1997): La era de la información. Economía, sociedad y cultura, Madrid, Alianza Editorial.

CASTELLS, M., Fernández-Ardèvol, M., Linchuan-Qiu, J. and Sey, A. (2006). Mobile Communication and Society. A Global Perspective, Cambridge, MIT Press.

CORTÉS, D. y GARZÓN, T. (2017): El ciberactivismo en las revoluciones posmodernas en Revista de Estudios en Seguridad Internacional, Vol. 3, n. 1, pp.103-125.

FLORIDA, R. (2009): Las ciudades creativas. Barcelona, Paidós.

FORMENTI, C. (2017): La variante populista. Lucha de clases en el neoliberalismo. Barcelona, El Viejo Topo.

GIL, H. y GUILLAUME, R. (2017): Redes de comunicación del movimiento $15 \mathrm{M}$ en Twitter en Revista Hispana para el Análisis de Redes Sociales, Vol. 28 (1), pp.136146.

GONZÁLEZ, J. (2012): Entre cultura(s) e cibercultur@(s). Incursoes e otras rotas nao lineares. Sao Bernardo do Campo, UMESP.

HAMELINK, C. (2000): The Ethics of Cyberspace, London, Sage.

INDOVINA, F. (2012): Del análisis del territorio al gobierno de la ciudad. Barcelona, Icaria.

BARBERO, J. M. (2002): Oficio de cartógrafo: Travesías latinoamericanas de la comunicación en la cultura. Fondo de Cultura Económica.

MISSENI, L.E. (2016): La institución de la ciberpolítica: gobernanza y Código, Revista Chasqui, Quito, n. 132, pp. 261-275. 
MODONESI, M. (Coord.) (2015): Movimientos subalternos, antagonistas y autónomos en México y América Latina. México, UNAM.

NAVARRO, V. (2003): Crítica del concepto de Capital Social, en Sistema. Revista de Ciencias Sociales, n. 172, pp.27-36.

NEUMAN, M.I. (2008): La apropiación tecnológica como práctica de resistencia y negociación en la globalización. IX Congreso Latinoamericano de Investigadores de la Comunicación. México.

PADILLA, M. (2012): El kit de la lucha en internet. Madrid,Traficantes de Sueños.

REGUILLO, R. (2017): Paisajes insurrectos. Jóvenes, redes y revueltas en el otoño civilizatorio. Madrid, NED EDICIONES.

RODRÍGUEZ, R. y MARTíNEZ, F. (2016): Poder e Internet. Un análisis crítico de la red. Madrid, Cátedra.

SABARIEGO, J. (2018): Los derechos humanos en la era Twitter: la tecnopolítica de los Recientes Movimientos Sociales Globales. $70^{\circ}$ Aniversario de la Declaración Universal de Derechos Humanos: la Protección Internacional de los Derechos Humanos en cuestión, Vol. 2, pp. 301-312. Valencia, Editorial Tirant lo Blanch.

SÁNCHEZ, J.M. (2016): La red como espacio para la militancia política: tecnología y participación en campaña electoral. Communication \& Society, Vol. 29 (3), pp. 33-47.

SIERRA, F. (2010a): Capitalismo Cognitivo y Sociedad de la Información. La deriva privatista de la UE en Susana SEL (Coord.). Políticas de Comunicación en el capitalismo contemporáneo. América Latina y sus encrucijadas, Buenos Aires, Ediciones CLACSO., pp. 233-254.

SIERRA, F. (2010b): Ciudadanía, comunicación y gobernanza local. Consideraciones para una nueva política de lo común en SIERRA, F., GARCÍA, J.A., RAMOS, M.C. y DEL BIANCO, N. (Orgs.) (2010): Políticas de Comunicaçao e da Cultura: Contribuçoes acadêmicas e intervencao social, Brasília, Casa das Musas/INTERCOM, PP. 57-74.

(2019): Ciudadanía Digital. Madrid, Biblioteca Nueva.

SIERRA, F. (Coord.) (2013): Ciudadanía, Tecnología y Cultura. Nodos conceptuales para pensar la nueva mediación digital, Barcelona, Gedisa. 
SIERRA, F. y GRAVANTE, T. (2012): Apropiación tecnológica y mediación. Líneas y fracturas para pensar otra comunicación posible en ENCINA, J. y ÁVILA, M.A. (Eds). Autogestión de la vida cotidiana, Sevilla, UNILCO, pp.130-138.

(2017). Tecnopolítica en América Latina y el Caribe. Salamanca, Comunicación Social Ediciones y Publicaciones

VIZER, E. (2003): La trama invisible de la vida social. Buenos Aires, La Crujía. 